Revista Verde de Agroecologia e Desenvolvimento Sustentável

http://www.gvaa.com.br/revista/index.php/RVADS

ARTIGO CIENTÍFICO

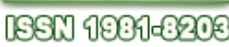

DOI: http://dx.doi.org/10.18378/rvads.v10i3.3315

\title{
Aspectos germinativos de cultivares de girassol armazenadas submetidos a estresse térmico
}

\section{Germinal aspects of stored sunflower cultivars subjected to heat stress}

\author{
Renato Leandro Costa Nunes $^{1^{*}}$, Alexandre Bosco de Oliveira ${ }^{2}$, Tatiana Maria da Silva ${ }^{3}$, André Luís da Silva Parente \\ Nogueira ${ }^{4}$ Maria Neurilan Costa Silva ${ }^{5}$
}

\begin{abstract}
Resumo: Objetivou-se avaliar a germinação de sementes de diferentes cultivares de girassol armazenadas sob condições de estresse térmico. O trabalho foi desenvolvido no Laboratório de Análise de Sementes do Departamento de Fitotecnia da Universidade Federal do Ceará (UFC), em Fortaleza - CE. Para a avaliação da influência da temperatura o experimento foi disposto em delineamento inteiramente casualizado em esquema fatorial $(20$ x 5), sendo vinte cultivares de girassol e cinco regimes de temperaturas $\left(25,0 ; 27,5 ; 30,0 ; 32,5\right.$ e $\left.35,0^{\circ} \mathrm{C}\right)$ e, respectivamente, conduzido quatro repetições. Foram avaliadas as seguintes variáveis: média da matéria fresca por plântula; média da matéria seca por plântula tempo médio de germinação; índice de velocidade de germinação; porcentagem de germinação. Os resultados foram comparados entre si pelo teste de SkottKnott a 5\% de probabilidade. A avaliação de estresse térmico promovido nas sementes de girassol, permitiram discriminar o híbrido Olissun como o que apresentou as melhores características em termos de qualidade fisiológica para superação de estresse térmico em comparação aos demais cultivares avaliados.
\end{abstract}

Palavras-chave: Helianthus annuus L. Temperatura. Estresse abiótico.

\begin{abstract}
This study aimed to evaluate the germination of seeds of different cultivars of sunflower under conditions of heat stress. The study was conducted in the Plant Science Department of the Seed Analysis Laboratory of the Federal University of Ceará (UFC) in Fortaleza-CE. For the evaluation of the influence of temperature, the experiment was arranged in a completely randomized design in a factorial design $(20 \times 5)$, and twenty varieties of sunflower five temperature regimes $(25.0,27.5,30.0$, 32.5 and $35,0^{\circ} \mathrm{C}$ ) and, respectively, conducted four replications. The following variables were evaluated: mean fresh weight per plant; average dry matter per plant average time of germination; germination speed index; germination percentage. The results were compared by Skott-Knott test at 5\% probability. The evaluation of thermal stress caused in sunflower seeds, allowed to discriminate the hybrid Olissun as the one with the best characteristics in terms of physiological quality to overcome heat stress compared to other cultivars evaluated.
\end{abstract}

Keywords: Helianthus annuus L. Temperature. Abiotic stress.

\footnotetext{
*Autor para correspondência

Recebido para publicação em 09/03/2015; aprovado em 15/07/2015

${ }^{1}$ Mestre em Fitotecnia, Universidade Federal do Ceará, renatoleandro.ce@ hotmail.com

${ }^{2}$ Dr. Professor, no PPG em Fitotecnia, Universidade Federal do Ceará, aleufc@ gmail.com

${ }^{3}$ Mestre em Fitotecnia, Universidade Federal do Ceará, tatianad.silva@ hotmail.com

${ }^{4}$ Aluno de Graduação, Universidade Federal do Ceará, andre_nogueira18@ @otmail.com

${ }^{5}$ Aluno de Graduação, Universidade Federal do Ceará, marianeuri@ hotmail.com
} 


\section{INTRODUÇÃO}

O girassol é cultivado nos cinco continentes, tendo grande importância na economia mundial (SILVA et al., 2012), sendo a Ucrânia, Rússia, Estados Unidos, Argentina e Turquia, nesta ordem, os principais produtores mundiais de seus grãos (USDA, 2013; CONAB, 2013). Segundo (NOBRE et al., 2011) o girassol responde por cerca de $13 \%$ de todo o óleo vegetal produzido no mundo.

O Brasil ainda ocupa uma posição pouco expressiva no que diz respeito à produção de girassol, entretanto, nos últimos anos tem sido significativo o incremento de áreas destinadas à sua exploração (BACKES et al., 2008). O crescimento da cultura em relação à área plantada está em ascendência (CONAB, 2005), prova disso é que a produção de girassol no Brasil ocupa atualmente uma área de aproximadamente $143,5 \mathrm{mil}$ hectares, apresentando um aumento expressivo de $104,7 \%$, se comparado com o da safra passada concentrando-se, sobretudo nos estados de Mato Grosso e Minas Gerais (CONAB, 2014).

O semiárido nordestino apresenta grandes potencialidades econômicas para o desenvolvimento da cultura do girassol, embora esta seja pouco cultivada na região em relação a outros estados da federação.

$\mathrm{O}$ pouco cultivo pode estar relacionado principalmente à reduzida quantidade de informação, principalmente de pesquisas, geradas pelos setores da sociedade responsáveis por realizá-las e divulgar os resultados obtidos. Sendo assim, tendo em vista a necessidade de estudos que envolvam a tecnologia de cultivo do girassol, é importante o desenvolvimento de trabalhos que estudem o comportamento germinativo de sementes desta espécie em condições edafoclimáticas de regiões em que se pretende cultivá-las.

A germinação só ocorre dentro de determinados limites de temperatura, nestes existe uma temperatura ou faixa considerada ótima, na qual ocorre a máxima germinação no menor período de tempo possível (CARVALHO; NAKAGAWA, 2000). Assim, a temperatura caracteriza-se por influenciar diretamente a porcentagem de germinação, afetando tanto a velocidade de absorção de água quanto suas reações bioquímicas (AMATO et al., 2007). Em consequência, afeta tanto a velocidade quanto a uniformidade de germinação, com influência direta na germinação total (CARVALHO; NAKAGAWA, 2000).

No entanto, a literatura é carente de informações sobre sementes híbridas e cultivares armazenadas por longo tempo, especialmente girassol, em relação aos testes de germinação com diferentes temperaturas, estresse salino e hídrico. Com base nessas considerações, objetivou-se estudar os aspectos germinativos de cultivares de girassol armazenadas submetidos a estresse térmico.

\section{MATERIAL E MÉTODOS}

Foram utilizados 20 cultivares de girassol, sendo: Aguará 04, Aguará B5, Aguará 06, BRS 321, BRS 323, BRS 324, Charrua P, CF 101, Helio 250, Helio 251, Helio 353K, Helio 358L, Helio 360, Neon, Olissun, Paraíso 65R, Paraíso T55, Paraíso 103S, Zenit e Embrapa 122, cujas sementes foram doadas pela empresa Petrobrás Biocombustível, em julho de 2013.

Antes do acondicionamento das sementes em câmara fria, as sementes foram armazenadas em ambiente natural por quatro meses. Em seguida foi realizada a assepsia dessas através da imersão em hipoclorito de sódio a 5\% durante 15 min, sendo posteriormente lavadas em água corrente e água destilada. Para garantir a assepsia das sementes, as mesmas foram lavadas com uma solução de fungicida a $0,4 \%$ (nistatina $^{\circledR}$ ) para evitar o ataque de fungos.

Após a assepsia das sementes, os híbridos foram acondicionados em sacos de polietileno e encaminhados para o Laboratório de Análise de Sementes do Departamento de Fitotecnia, da Universidade Federal do Ceará (UFC), em Fortaleza - CE, onde posteriormente, foram armazenadas em câmara fria $\left( \pm 12^{\circ} \mathrm{C}\right.$ e $\left.50 \%\right)$ até a instalação do experimento realizado no mês de julho de 2014

$\mathrm{O}$ efeito do fator abiótico (temperatura) foi verificado em um experimento disposto em delineamento inteiramente casualizado em esquema fatorial $20 \times 5$, cinco regimes de temperatura e vinte cultivares, com quatro repetições de 50 sementes.

Para o teste de germinação, as sementes foram postas para germinar em rolos de papel toalha, tipo "germitest", previamente umedecido com água destilada com volume equivalente a 2,5 vezes o peso do papel seco, sendo estes acondicionados em câmaras de germinação reguladas nas temperaturas de 25,$0 ; 27,5 ; 30,0 ; 32,5$ e $35,0{ }^{\circ} \mathrm{C}$. Dez dias aposta à semeadura foi realizado a porcentagem de germinação considerando as sementes com emissão da radícula com tamanho igual ou superior a $2 \mathrm{~mm}$. Em conjunto com o teste de germinação, foi realizado índice de velocidade de germinação (MAGUIRE, 1962), tempo médio de germinação (LABORIAU, 1977) e massa fresca e seca de plântulas normais, coletadas ao final do teste de germinação.

Para fins de análise estatística, os dados obtidos foram submetidos a análise de variância (ANOVA) e teste de SkottKnott (5\%) para comparação das médias. Para auxílio nas análises estatísticas utilizou-se o programa Sisvar 5.3 (FERREIRA, 2010).

\section{RESULTADOS E DISCUSSÃO}

O resumo da análise de variância mostra que houve diferença para os fatores avaliados de forma isolada e conjunta, para todas as características avaliadas (Tabela 1).

Observa-se na Tabela 1, que as temperaturas influenciaram na porcentagem de germinação (PG) dos diferentes cultivares avaliados, com destaque sendo feito para o híbrido Olissun que apresentou o melhor desempenho, com $85 \%$ de germinação a temperatura de $30^{\circ} \mathrm{C}$. A temperatura constante de $30^{\circ} \mathrm{C}$ também possibilitou a obtenção de resultados positivos quanto a germinação de sementes oleaginosas de pinhão-manso (MARTINS et al., 2008). Igualmente as sementes de niger atingiram maiores níveis de germinação sob temperaturas de até $30^{\circ} \mathrm{C}$, sendo intolerantes a temperaturas abaixo de $10^{\circ} \mathrm{C}$ (GORDIN et al., 2014). 
Tabela 1. Resumo da ANOVA para as variáveis porcentagem de germinação (PG), índice de velocidade de germinação (IVG), tempo médio de germinação (TMG) matéria fresca (MF) e matéria seca por plântula (MS) de sementes de diferentes cultivares de girassol submetidos a diferentes níveis de temperatura

\begin{tabular}{|c|c|c|c|c|c|c|}
\hline \multirow{2}{*}{ FV } & \multicolumn{6}{|c|}{$\mathrm{QM}$} \\
\hline & GL & PG (\%) & IVG & TMG & MF (g/plântula) & MS (g/plântula) \\
\hline Cultivares (C) & 19 & $5114,19^{* * *}$ & $184,39^{\text {** }}$ & $45,91^{* * *}$ & $0,32^{* *}$ & $8,0 \times 10^{-3 * *}$ \\
\hline Temperatura (T) & 4 & $2037,93^{* *}$ & $66,93^{* * *}$ & $5,13^{* *}$ & $0,16^{* *}$ & $2,0 \times 10^{-3^{*}}$ \\
\hline (C) $\mathrm{x}(\mathrm{T})$ & 76 & $201,59^{* *}$ & $8,792^{* *}$ & $2,26^{* *}$ & $0,02^{* *}$ & $1,0 \times 10^{-3 * *}$ \\
\hline Erro & 300 & 20,95 & 0,56 & 1,17 & 0,01 & $1 \times 10^{-3}$ \\
\hline $\mathrm{CV}(\%)$ & - & 31,35 & 29,66 & 43,94 & 39,91 & 79,24 \\
\hline Média geral & - & 14,60 & 2,52 & 2,46 & 0,18 & 0,03 \\
\hline
\end{tabular}

significância a 1\%, " significância a 5\%, ambos pelo teste de Scott Knott.

A temperatura onde ocorre o maior índice de geminação no menor período de tempo é considerada como temperatura ótima, sendo recomendada para condução do teste de germinação da referida espécie (CARVALHO; NAKAGAWA, 2000).

Os melhores resultados para a porcentagem de germinação das sementes foram observados, em geral, com a temperatura de $30^{\circ} \mathrm{C}$, onde a partir disto a PG foi fortemente afetada à medida que se elevou a temperatura, consequentemente verificando a não germinação das sementes de sete dos vinte cultivares avaliados sob a temperatura de $35^{\circ} \mathrm{C}$ (Tabela 2).

Tal resultado deve-se ao fato de que temperaturas elevadas interferem de forma negativa nas reações metabólicas responsáveis por desencadear o processo germinativo das sementes, podendo inclusive paralisar totalmente o processo de germinação. Sob temperatura de $35^{\circ}$ C, seis dias após a instalação o início, as sementes de girassol apresentavam-se com o tegumento escurecido, com exsudato de aspecto gelatinoso no meio germinativo. Sementes de pinhão-manso apresentaram comportamento irregular ao mesmo nível de temperatura, sendo que as plântulas obtidas nesta condição apresentaram aspecto anormal (MARTINS et al., 2008).

Para índice de velocidade de germinação (IVG), verificou-se que o valor em destaque em relação aos híbridos e cultivar avaliados foi de 19,07 representado pelo híbrido Olissun sob temperatura constante de $30^{\circ} \mathrm{C}$, (Tabela 3). (PASCUALI et al., 2012) apresentaram resultados similares em relação a temperatura, onde sementes de pinhão manso, obtiveram valor superior de IVG a $30^{\circ} \mathrm{C}$.

Tabela 2. Porcentagem de germinação (PG) de sementes de 20 cultivares de girassol submetidos a diferentes níveis de temperatura 25,$0 ; 27,5 ; 30,0 ; 32,5$ e $35,0^{\circ} \mathrm{C}$, constantes.

\begin{tabular}{|c|c|c|c|c|c|}
\hline \multirow{3}{*}{ Híbridos } & \multicolumn{5}{|c|}{ Temperatura } \\
\hline & $25^{\circ} \mathrm{C}$ & $27,5^{\circ} \mathrm{C}$ & $30^{\circ} \mathrm{C}$ & $32,5^{\circ} \mathrm{C}$ & $35^{\circ} \mathrm{C}$ \\
\hline & \multicolumn{5}{|c|}{ PG (\%) } \\
\hline Aguará 04 & $0 \mathrm{f} \mathrm{A}$ & $0 \mathrm{f} \mathrm{A}$ & $0 \mathrm{f} \mathrm{A}$ & 0 e $\mathrm{A}$ & $0 \mathrm{dA}$ \\
\hline Paraíso 65R & $0 \mathrm{f} \mathrm{A}$ & $0 \mathrm{f} \mathrm{A}$ & $0 \mathrm{f} \mathrm{A}$ & 0 e $\mathrm{A}$ & $0 \mathrm{dA}$ \\
\hline Paraíso 103S & $1 \mathrm{f} \mathrm{A}$ & $0 \mathrm{f} \mathrm{A}$ & $0 \mathrm{f} \mathrm{A}$ & 0 e $A$ & $0 \mathrm{dA}$ \\
\hline Zenit & $0 \mathrm{f} \mathrm{A}$ & $0 \mathrm{f} \mathrm{A}$ & $0 \mathrm{f} \mathrm{A}$ & 1 e $A$ & $0 \mathrm{dA}$ \\
\hline Helio 360 & $0 \mathrm{f} \mathrm{A}$ & $0 \mathrm{f} \mathrm{A}$ & $1 \mathrm{f} \mathrm{A}$ & $1 \mathrm{e} A$ & $0 \mathrm{dA}$ \\
\hline Neon & $0 \mathrm{f} \mathrm{A}$ & $0 \mathrm{f} \mathrm{A}$ & $2 \mathrm{f} \mathrm{A}$ & 5 e $A$ & $2 \mathrm{dA}$ \\
\hline Helio $353 \mathrm{~K}$ & $2 \mathrm{f} \mathrm{A}$ & $1 \mathrm{f} \mathrm{A}$ & $4 \mathrm{f} \mathrm{A}$ & 4 e $A$ & $1 \mathrm{~d} \mathrm{~A}$ \\
\hline BRS 324 & 10 e $\mathrm{A}$ & $4 \mathrm{f} \mathrm{B}$ & $1 \mathrm{fB}$ & 4 e B & $0 \mathrm{~d} \mathrm{~B}$ \\
\hline BRS 321 & 13 e $\mathrm{A}$ & $7 \mathrm{f} \mathrm{B}$ & $6 \mathrm{fB}$ & 2 e B & $0 \mathrm{~d} \mathrm{~B}$ \\
\hline Helio 251 & 14 e B & $22 \mathrm{~d} \mathrm{~A}$ & 24 e A & $12 \mathrm{~d} \mathrm{~B}$ & $7 \mathrm{c} \mathrm{B}$ \\
\hline Helio 250 & $20 \mathrm{~d} \mathrm{~A}$ & 10 e $B$ & 23 e $A$ & $21 \mathrm{c} \mathrm{A}$ & $4 \mathrm{c} \mathrm{C}$ \\
\hline Helio 358L & $18 \mathrm{~d} \mathrm{~A}$ & $19 \mathrm{~d} \mathrm{~A}$ & 23 e $A$ & $13 \mathrm{~d} \mathrm{~B}$ & $9 \mathrm{c} \mathrm{B}$ \\
\hline BRS 323 & $21 \mathrm{~d} \mathrm{~B}$ & 15 e $\mathrm{B}$ & $34 \mathrm{~d} \mathrm{~A}$ & $17 \mathrm{~d} \mathrm{~B}$ & $18 \mathrm{~b} \mathrm{~B}$ \\
\hline Embrapa 122 & $17 \mathrm{~d} \mathrm{~B}$ & $28 \mathrm{c} \mathrm{A}$ & $31 \mathrm{~d} \mathrm{~A}$ & 23 c B & $3 \mathrm{c} \mathrm{C}$ \\
\hline Aguará B5 & $22 \mathrm{~d} \mathrm{~A}$ & $5 \mathrm{fB}$ & 24 e A & $23 \mathrm{c} \mathrm{A}$ & $13 \mathrm{~b} \mathrm{~B}$ \\
\hline Aguará 06 & 30 c A & 33 с A & 26 e A & $16 \mathrm{~d} \mathrm{~B}$ & $4 \mathrm{c} \mathrm{C}$ \\
\hline Charrua P & $33 \mathrm{c} \mathrm{A}$ & $27 \mathrm{c} \mathrm{A}$ & $70 \mathrm{~d} \mathrm{~A}$ & $33 \mathrm{~b} \mathrm{~A}$ & $15 \mathrm{~b} \mathrm{~B}$ \\
\hline Paraíso T55 & $18 \mathrm{~d} \mathrm{C}$ & $33 \mathrm{c} \mathrm{B}$ & $50 \mathrm{~b} \mathrm{~A}$ & $30 \mathrm{~b} \mathrm{~B}$ & $16 \mathrm{~b} \mathrm{C}$ \\
\hline CF 101 & $40 \mathrm{~b} \mathrm{~A}$ & $40 \mathrm{~b} \mathrm{~A}$ & $41 \mathrm{c} \mathrm{A}$ & $29 \mathrm{~b} \mathrm{~B}$ & $6 \mathrm{c} \mathrm{C}$ \\
\hline Olissun & 58 a D & 78 a B & 85 a A & 70 a $C$ & 33 a $E$ \\
\hline
\end{tabular}

Letras iguais, maiúsculas nas linhas e minúsculas nas colunas, não diferem entre si pelo teste de Scott Knott5\% de probabilidade de erro. 
Tabela 3. Índice de velocidade de germinação (IVG) e tempo médio de germinação (TMG) de sementes de 20 cultivares de girassol submetidos a diferentes níveis de temperatura 25,$0 ; 27,5 ; 30,0 ; 32,5$ e $35,0^{\circ} \mathrm{C}$, constantes.

\begin{tabular}{|c|c|c|c|c|c|}
\hline \multirow{3}{*}{ Híbridos } & \multicolumn{5}{|c|}{ Tratamentos } \\
\hline & $25^{\circ} \mathrm{C}$ & $27,5^{\circ} \mathrm{C}$ & $30^{\circ} \mathrm{C}$ & $32,5^{\circ} \mathrm{C}$ & $35^{\circ} \mathrm{C}$ \\
\hline & \multicolumn{5}{|c|}{ IVG } \\
\hline Aguará 04 & 0,000 e $\mathrm{A}$ & 0,000 e $\mathrm{A}$ & $0,000 \mathrm{f} \mathrm{A}$ & $0,000 \mathrm{dA}$ & $0,000 \mathrm{~d} \mathrm{~A}$ \\
\hline Paraíso 65R & 0,000 e $A$ & 0,000 e $\mathrm{A}$ & $0,000 \mathrm{fA}$ & $0,000 \mathrm{~d} A$ & $0,000 \mathrm{~d} \mathrm{~A}$ \\
\hline Paraíso 103S & 0,050 e $A$ & 0,000 e $\mathrm{A}$ & $0,000 \mathrm{fA}$ & $0,000 \mathrm{~d} \mathrm{~A}$ & $0,000 \mathrm{~d} \mathrm{~A}$ \\
\hline Zenit & 0,000 e $A$ & 0,000 e $A$ & $0,000 \mathrm{f} \mathrm{A}$ & $0,188 \mathrm{~d} A$ & $0,000 \mathrm{~d} \mathrm{~A}$ \\
\hline Helio 360 & 0,000 e $\mathrm{A}$ & 0,000 e $A$ & $0,167 \mathrm{f} \mathrm{A}$ & $0,208 \mathrm{~d} A$ & $0,000 \mathrm{~d} \mathrm{~A}$ \\
\hline Neon & 0,000 e $A$ & 0,000 e $A$ & $0,354 \mathrm{f} \mathrm{A}$ & $0,807 \mathrm{~d} \mathrm{~A}$ & $0,217 \mathrm{~d} \mathrm{~A}$ \\
\hline Helio $353 \mathrm{~K}$ & 0,248 e $\mathrm{A}$ & 0,036 e $\mathrm{A}$ & $0,619 \mathrm{f} \mathrm{A}$ & $0,583 \mathrm{~d} \mathrm{~A}$ & $0,042 \mathrm{~d} \mathrm{~A}$ \\
\hline BRS 324 & $1,994 \mathrm{~d} \mathrm{~A}$ & 0,663 e $B$ & $0,146 \mathrm{f} \mathrm{B}$ & $0,529 \mathrm{~d} \mathrm{~B}$ & $0,000 \mathrm{~d} \mathrm{~B}$ \\
\hline BRS 321 & $2,292 \mathrm{~d} \mathrm{~A}$ & 0,780 е $\mathrm{B}$ & $0,808 \mathrm{fB}$ & $0,375 \mathrm{~d} \mathrm{~B}$ & $0,000 \mathrm{~d} \mathrm{~B}$ \\
\hline Helio 251 & $2,162 \mathrm{~d} \mathrm{~B}$ & $2,483 \mathrm{~d} \mathrm{~B}$ & 3,542 e $A$ & $2,046 \mathrm{c} \mathrm{B}$ & $1,092 \mathrm{c} \mathrm{C}$ \\
\hline Helio 250 & $2,961 \mathrm{c} \mathrm{A}$ & 1,235 e $B$ & 3,187 e $A$ & $2,721 \mathrm{c} \mathrm{A}$ & $0,425 \mathrm{~d} \mathrm{~B}$ \\
\hline Helio 358L & $3,634 \mathrm{c} \mathrm{B}$ & $2,357 \mathrm{~d} \mathrm{C}$ & $4,812 \mathrm{~d} \mathrm{~A}$ & 2,259 c C & $1,521 \mathrm{c} \mathrm{C}$ \\
\hline BRS 323 & $3,871 \mathrm{c} \mathrm{B}$ & $2,498 \mathrm{~d} \mathrm{~B}$ & $7,167 \mathrm{~b} \mathrm{~A}$ & 3,296 c B & $3,083 \mathrm{~b} \mathrm{~B}$ \\
\hline Embrapa 122 & $2,091 \mathrm{~d} \mathrm{~B}$ & $3,968 \mathrm{c} \mathrm{A}$ & 3,499 e A & $2,721 \mathrm{c} \mathrm{B}$ & $0,435 \mathrm{~d} \mathrm{C}$ \\
\hline Aguará B5 & $3,346 \mathrm{c} \mathrm{B}$ & 0,663 e $\mathrm{D}$ & $4,396 \mathrm{~d} \mathrm{~A}$ & 4,746 b A & $2,371 \mathrm{~b} \mathrm{C}$ \\
\hline Aguará 06 & $5,525 \mathrm{~b} \mathrm{~A}$ & $4,808 \mathrm{c} \mathrm{A}$ & $5,153 \mathrm{~d} \mathrm{~A}$ & $3,304 \mathrm{c} \mathrm{B}$ & $0,688 \mathrm{c} \mathrm{C}$ \\
\hline Charrua P & $6,053 \mathrm{~b} \mathrm{~A}$ & 4,503 c B & 6,034 c A & 4,609 b B & $2,240 \mathrm{~b} \mathrm{C}$ \\
\hline Paraíso T55 & 3,488 с C & 4,888 c B & $7,964 \mathrm{~b} \mathrm{~A}$ & 4,892 b B & $2,771 \mathrm{~b} \mathrm{C}$ \\
\hline CF 101 & $6,375 \mathrm{~b} \mathrm{~A}$ & $5,937 \mathrm{~b} \mathrm{~A}$ & $6,569 \mathrm{c} \mathrm{A}$ & 4,929 b B & $1,083 \mathrm{c} \mathrm{C}$ \\
\hline Olissun & 12,396 a C & 19,073 a $A$ & 17,040 a $B$ & 10,453 a $D$ & 5,396 a E \\
\hline
\end{tabular}

Letras iguais, maiúsculas nas linhas e minúsculas nas colunas, não diferem entre si pelo teste de Scott Knott5\% de probabilidade de erro.

Para a característica tempo médio de germinação (TMG), a cultivar Embrapa 122 apresentou o maior valor, 5,27 , também sob $30^{\circ} \mathrm{C}$ de temperatura. Essa superioridade da Embrapa 122 pode ter ocorrido devido a características intrínsecas da cultivar, como maior rusticidade por exemplo, o que possibilita melhor adaptação desta a condições adversas (ALVES et al., 2013). De acordo com Gordin et al., (2012) estudando a germinação de sementes de niger (Guizotia abyssinica Cass.), outra espécie oleaginosa, temperaturas de $20-30^{\circ} \mathrm{C}$ e $30^{\circ} \mathrm{C}$ proporcionaram valores de tempo médio de germinação elevados.

Analisando as respostas referentes à Tabela 3 , observase que os menores índices de velocidade de germinação foram observados em sementes postas para germinar a $35^{\circ} \mathrm{C}$. Estudos realizados com sementes de pinhão manso também obtiveram valores inferiores em temperaturas de $35^{\circ} \mathrm{C}$ (PASCUALI et al., 2012). Assim o conceito de temperatura ótima de germinação, deve considerar outros aspectos como tempo médio de germinação e índice de velocidade de emergência e, não apenas a porcentagem de germinação. Para a massa fresca (MF) por plântula, verifica-se na Tabela 4 que os menores valores de MF, em geral, foram obtidos sob a temperatura máxima de $35^{\circ} \mathrm{C}$, à exceção dos híbridos Helio 358L e BRS 323. Temperaturas altas alteram a velocidade de absorção de água e a velocidade das reações metabólicas necessárias para a germinação e, consequentemente, a produção de biomassa na plântula. $\mathrm{O}$ maior valor de $\mathrm{MF}$ ocorreu sob a temperatura de $25^{\circ} \mathrm{C}(0,51 \mathrm{~g})$, para o híbrido $\mathrm{CF} 101$, porém foi à temperatura de $30^{\circ} \mathrm{C}$ sobre as sementes que proporcionou uma melhor produção de matéria fresca. Isto se deve a influência da temperatura na aceleração do metabolismo das sementes, promovendo reações bioquímicas nas quais as macromoléculas presentes no tecido de reserva são hidrolisadas, transportadas e ressintetizadas no eixo embrionário. Em plântulas de niger a variável matéria fresca, apresentou resultados estatisticamente superiores sob temperaturas de 15,25 e $20-30^{\circ} \mathrm{C}$ (GORDIN et al., 2014). O acúmulo de biomassa nas plântulas é decorrente de uma série de reações enzimáticas, as quais se processam lenta ou rapidamente seja em baixas ou altas temperaturas. Segundo Zanella et al., (2006), para qualquer reação química, existe uma temperatura ótima na qual o processo se realiza mais rápida e eficientemente.

Ainda na Tabela 4 os menores valores de MS por plântula também foram obtidos para a temperatura máxima $\left(35^{\circ} \mathrm{C}\right)$. Podemos observar que a elevação da temperatura afeta o poder de germinação das sementes e, consequentemente, a matéria seca das plântulas. Apesar de menor, a temperatura de $20^{\circ} \mathrm{C}$ proporcionou os menores acúmulos de massa seca de plântulas de niger (GORDIN et al., 2014).

Observou-se ainda para MS que o valor em destaque em relação aos cultivares avaliados foi $0,08 \mathrm{~g}$ representado pelo híbrido CF 101, sob temperatura de $32,5^{\circ} \mathrm{C}$. Em temperaturas menores, as plântulas de niger, para a massa seca, obtiveram os maiores conteúdos na temperatura de $15^{\circ} \mathrm{C}$ (GORDIN et al., 2014). Portanto, a temperatura afetou o peso das plântulas em matéria fresca e seca, mostrando que as sementes possuem um melhor desempenho dentro de uma faixa determinada de temperatura, que é típica para cada híbrido e cultivar. 
Tabela 4. Matéria fresca (MF) e seca (MS) de plântulas de girassol oriundas de sementes de 20 cultivares de girassol submetidos a diferentes níveis de temperatura 25,$0 ; 27,5 ; 30,0 ; 32,5$ e $35,0^{\circ} \mathrm{C}$, constantes.

\begin{tabular}{|c|c|c|c|c|c|}
\hline \multirow{3}{*}{ Sementes } & \multicolumn{5}{|c|}{ Temperatura } \\
\hline & $25^{\circ} \mathrm{C}$ & $27,5^{\circ} \mathrm{C}$ & $30^{\circ} \mathrm{C}$ & $32,5^{\circ} \mathrm{C}$ & $35^{\circ} \mathrm{C}$ \\
\hline & \multicolumn{5}{|c|}{ MF (g/plântula) } \\
\hline Aguará 04 & $0,00 \mathrm{~d} \mathrm{~A}$ & $0,00 \mathrm{~d} \mathrm{~A}$ & $0,00 \mathrm{dA}$ & $0,00 \mathrm{dA}$ & $0,00 \subset \mathrm{A}$ \\
\hline Paraíso 65R & $0,00 \mathrm{~d} \mathrm{~A}$ & $0,00 \mathrm{~d} \mathrm{~A}$ & $0,00 \mathrm{~d} \mathrm{~A}$ & $0,00 \mathrm{~d} \mathrm{~A}$ & $0,00 \subset \mathrm{A}$ \\
\hline Paraíso 103S & $0,08 \mathrm{~d} A$ & $0,00 \mathrm{~d} A$ & $0,00 \mathrm{~d} \mathrm{~A}$ & $0,00 \mathrm{dA}$ & $0,00 \subset \mathrm{A}$ \\
\hline Zenit & $0,00 \mathrm{~d} B$ & $0,00 \mathrm{~d} B$ & $0,00 \mathrm{~d} B$ & 0,11 с A & 0,00 c B \\
\hline Helio 360 & $0,00 \mathrm{~d} A$ & $0,00 \mathrm{~d} A$ & $0,04 \mathrm{~d} A$ & 0,07 c A & $0,00 \mathrm{cA}$ \\
\hline Neon & $0,00 \mathrm{~d} \mathrm{~B}$ & $0,00 \mathrm{~d} \mathrm{~B}$ & 0,14 c A & 0,15 c A & 0,06 c B \\
\hline Helio $353 \mathrm{~K}$ & $0,07 \mathrm{~d} \mathrm{~B}$ & $0,02 \mathrm{~d} \mathrm{~B}$ & $0,11 \mathrm{c} \mathrm{A}$ & $0,20 \mathrm{~b} \mathrm{~A}$ & 0,01 c B \\
\hline BRS 324 & 0,18 с A & $0,24 \mathrm{c} \mathrm{A}$ & $0,04 \mathrm{~d} B$ & 0,11 с B & $0,00 \mathrm{c} \mathrm{B}$ \\
\hline BRS 321 & 0,26 с A & 0,18 с A & 0,14 c A & 0,10 с B & 0,00 c C \\
\hline Helio 251 & $0,25 \mathrm{cA}$ & 0,22 с A & 0,29 a $A$ & $0,20 \mathrm{~b} \mathrm{~A}$ & 0,19 a $\mathrm{A}$ \\
\hline Helio 250 & $0,30 \subset \mathrm{A}$ & $0,28 \subset \mathrm{A}$ & 0,30 a $A$ & 0,28 a $A$ & $0,11 \mathrm{~b} \mathrm{~B}$ \\
\hline Helio 358L & 0,29 с A & $0,35 \mathrm{~b} \mathrm{~A}$ & $0,20 \mathrm{~b} \mathrm{~A}$ & 0,30 a $\mathrm{A}$ & 0,26 a $A$ \\
\hline BRS 323 & 0,23 c A & 0,26 c A & $0,17 \mathrm{~b}$ B & 0,31 a $\mathrm{A}$ & 0,19 a $\mathrm{B}$ \\
\hline Embrapa 122 & 0,28 с A & $0,35 \mathrm{~b} \mathrm{~A}$ & $0,24 \mathrm{~b} \mathrm{~A}$ & 0,27 a $\mathrm{A}$ & $0,12 \mathrm{~b} \mathrm{~B}$ \\
\hline Aguará B5 & $0,36 \mathrm{~b}$ B & 0,50 a $A$ & 0,26 a $\mathrm{C}$ & 0,34 a B & 0,22 a $\mathrm{C}$ \\
\hline Aguará 06 & $0,39 \mathrm{~b} \mathrm{~A}$ & $0,36 \mathrm{~b} \mathrm{~A}$ & 0,28 a $A$ & 0,31 a $A$ & 0,09 c B \\
\hline Charrua P & 0,29 с A & $0,29 \mathrm{~b} \mathrm{~A}$ & 0,32 a $A$ & 0,29 a $\mathrm{A}$ & 0,21 a $\mathrm{A}$ \\
\hline Paraíso T55 & 0,32 с A & $0,36 \mathrm{~b} \mathrm{~A}$ & 0,30 a $A$ & 0,31 a $A$ & 0,25 a A \\
\hline CF 101 & 0,51 a $A$ & 0,49 a $\mathrm{A}$ & 0,30 a B & 0,32 a $\mathrm{B}$ & $0,12 \mathrm{~b} \mathrm{C}$ \\
\hline Olissun & $0,38 \mathrm{~b} \mathrm{~A}$ & $0,31 \mathrm{~b} \mathrm{~A}$ & 0,29 a $A$ & 0,28 a $A$ & 0,24 a A \\
\hline Sementes & \multicolumn{5}{|c|}{ MS (g/plântula) } \\
\hline Aguará 04 & 0,00 с A & $0,00 \mathrm{~b} \mathrm{~A}$ & $0,00 \mathrm{c} \mathrm{A}$ & $0,00 \mathrm{~b} \mathrm{~A}$ & $0,00 \mathrm{~b} \mathrm{~A}$ \\
\hline Paraíso 65R & 0,00 с A & $0,00 \mathrm{~b} \mathrm{~A}$ & 0,00 с A & $0,00 \mathrm{~b} \mathrm{~A}$ & $0,00 \mathrm{~b} \mathrm{~A}$ \\
\hline Paraíso 103S & $0,00 \subset \mathrm{A}$ & $0,00 \mathrm{~b} \mathrm{~A}$ & 0,00 c A & $0,00 \mathrm{~b} \mathrm{~A}$ & $0,00 \mathrm{~b} \mathrm{~A}$ \\
\hline Zenit & $0,00 \mathrm{c} \mathrm{A}$ & $0,00 \mathrm{~b} \mathrm{~A}$ & 0,00 c A & $0,02 \mathrm{~b} \mathrm{~A}$ & $0,00 \mathrm{~b} \mathrm{~A}$ \\
\hline Helio 360 & 0,00 с B & $0,00 \mathrm{~b} \mathrm{~B}$ & 0,10 a $A$ & $0,01 \mathrm{~b} \mathrm{~B}$ & $0,00 \mathrm{~b} \mathrm{~B}$ \\
\hline Neon & $0,00 \subset \mathrm{A}$ & $0,00 \mathrm{~b} \mathrm{~A}$ & $0,01 \mathrm{c} \mathrm{A}$ & 0,03 a $\mathrm{A}$ & 0,03 a $A$ \\
\hline Helio $353 \mathrm{~K}$ & $0,01 \mathrm{cA}$ & $0,00 \mathrm{~b} \mathrm{~A}$ & 0,02 c A & 0,03 a $\mathrm{A}$ & $0,00 \mathrm{~b} \mathrm{~A}$ \\
\hline BRS 324 & $0,05 \mathrm{~b} \mathrm{~A}$ & 0,04 a $A$ & 0,02 c B & 0,05 a $\mathrm{A}$ & $0,01 \mathrm{~b} \mathrm{~B}$ \\
\hline BRS 321 & 0,11 a $\mathrm{A}$ & 0,03 a $\mathrm{B}$ & $0,04 \mathrm{~b}$ B & $0,02 \mathrm{~b} \mathrm{~B}$ & $0,00 \mathrm{~b} \mathrm{~B}$ \\
\hline Helio 251 & $0,04 \mathrm{~b} \mathrm{~A}$ & 0,04 a A & $0,05 \mathrm{~b} \mathrm{~A}$ & 0,04 a A & 0,05 a $\mathrm{A}$ \\
\hline Helio 250 & $0,04 \mathrm{~b} \mathrm{~A}$ & 0,04 a $A$ & $0,05 \mathrm{~b} \mathrm{~A}$ & 0,06 a $\mathrm{A}$ & 0,05 a $\mathrm{A}$ \\
\hline Helio 358L & $0,04 \mathrm{~b} \mathrm{~A}$ & 0,05 a $\mathrm{A}$ & $0,03 \mathrm{~b} \mathrm{~A}$ & 0,06 a $\mathrm{A}$ & 0,05 a $\mathrm{A}$ \\
\hline BRS 323 & $0,03 \mathrm{~b} \mathrm{~A}$ & 0,04 a $\mathrm{A}$ & 0,02 c A & 0,04 a $\mathrm{A}$ & 0,04 a A \\
\hline Embrapa 122 & $0,04 \mathrm{~b} \mathrm{~A}$ & 0,05 a $A$ & $0,03 \mathrm{~b} \mathrm{~A}$ & 0,06 a $\mathrm{A}$ & 0,04 a A \\
\hline Aguará B5 & $0,04 \mathrm{~b} \mathrm{~A}$ & 0,03 a $A$ & $0,04 \mathrm{~b} \mathrm{~A}$ & 0,05 a $\mathrm{A}$ & 0,05 a $A$ \\
\hline Aguará 06 & $0,05 \mathrm{~b} \mathrm{~A}$ & 0,05 a $\mathrm{A}$ & $0,04 \mathrm{~b} \mathrm{~A}$ & 0,04 a $\mathrm{A}$ & 0,04 a A \\
\hline Charrua P & $0,04 \mathrm{~b} \mathrm{~A}$ & 0,05 a $\mathrm{A}$ & $0,05 \mathrm{~b} \mathrm{~A}$ & 0,06 a $\mathrm{A}$ & 0,03 a $A$ \\
\hline Paraíso T55 & $0,03 \mathrm{~b} \mathrm{~A}$ & 0,05 a $\mathrm{A}$ & $0,04 \mathrm{~b} A$ & 0,06 a $\mathrm{A}$ & 0,04 a $\mathrm{A}$ \\
\hline CF 101 & $0,06 \mathrm{~b} \mathrm{~A}$ & 0,06 a $A$ & $0,05 \mathrm{~b} \mathrm{~A}$ & 0,08 a $\mathrm{A}$ & 0,05 a $\mathrm{A}$ \\
\hline Olissun & $0,04 \mathrm{~b} \mathrm{~A}$ & 0,04 a $\mathrm{A}$ & $0,03 \mathrm{~b} \mathrm{~A}$ & 0,04 a $\mathrm{A}$ & $0,02 \mathrm{~b} \mathrm{~A}$ \\
\hline
\end{tabular}

Letras iguais, maiúsculas nas linhas e minúsculas nas colunas, não diferem entre si pelo teste de Scott Knott5\% de probabilidade de erro.

\section{CONCLUSÕES}

A temperatura de $30^{\circ} \mathrm{C}$ apresentou os melhores resultados para as características avaliadas dos cultivares estudados.

A cultivar Olissun, destacou-se nas avaliações das variáveis citadas, sob a temperatura de $30^{\circ} \mathrm{C}$, em especial para porcentagem de germinação.

\section{REFERÊNCIAS}

ALVES, G.S.; TARTAGLIA, F.L.; ROSA, J.C.; LIMA, P.C.; CARDOSO, G.D.; BELTRÃO, N.E.M. Períodos de interferência das plantas daninhas na cultura do girassol em
Rondônia, R. Bras. Eng. Agríc. Ambiental, v.17, n.3, p.275282, 2013.

AMATO, A.L.P.; MAIA, F.C.; MAIA, M.S.; CAETANO, L.S.; SIMIONI, S.B.; CONTO, L.; BONINI FILHO, R.M. Estabelecimento de condições de luz e temperatura na germinação de sementes de amendoim forrageiro, Revista Brasileira de Sementes, Londrina, v. 29, n. 3, p. 61-66, 2007.

BACKES, R.L.; SOUZA, A.M.; BALBINOT JUNIOR, A.A; GALlOTTI, G.J.M.; BAVARESCO, A. Desempenho de cultivares de girassol em duas épocas de plantio de safrinha no Planalto Norte Catarinense, Scientia Agraria, v. 09, n. 01, p. 41-48, 2008. 
BEWLEY, J.D.; BLACK, M. Seeds: physiology of development and germination. 2.ed. New York: Plenum Press, 1994. 445p.

CARVALHO, N.M.; NAKAGAWA, J. Sementes: ciência, tecnologia e produção. 4. ed. Jaboticabal: Funep, 2000. 588 p.

CONAB - Companhia Nacional De Abastecimento. Acompanhamento da Safra Brasileira de Grãos 2004/2005Primeiro Levantamento - Junho/2005.

CONAB - Companhia Nacional De Abastecimento. Acompanhamento da Safra Brasileira de Grãos 2012/2013Levantamento - Fevereiro/2013.

CONAB - Companhia Nacional De Abastecimento. Acompanhamento da Safra Brasileira de Grãos 2013/2014Décimo Levantamento - Junho/2014.

FERREIRA, D.F. Sisvar - Sistema de análise de variância. Versão 5.3. Lavras-MG: UFLA, 2010.

GORDIN, C.R.B.; MARQUES, R.F.; MASETTO, T.E.; SCALON, S.P.Q. Germinação, biometria de sementes e morfologia de plântulas de GuizotiaabyssinicaCass., Revista Brasileira de Sementes, vol. 34, no 4 p. 619 - 627, 2012.

GORDIN, C.R.B.; MARQUES, R.F.; MASETTO, T.E.; SCALON, S.P.Q.; SOUZA, L.C.F. Temperaturas e disponibilidades hídricas do substrato na germinação de sementes de niger, Biosci. J., Uberlandia, v. 30, supplement 1, p. 112-118, 2014.

LABORIAU, L.G. Shift of the maximum temperature of Viceagraminea seeds following imbibition of deuterium oxide, Journal of Thermal Biology, v. 2, p. 111-114, 1977.
MAGUIRE, J.D. Speed of germination-aid in selection and evaluation for seedling emergence and vigor, Crop Science, Madison, v. 2, n. 1, p. 176-177, 1962.

MARTINS, C.C.; MACHADO, C.G.; CAVASINI, R. Temperatura e substrato para o teste de germinação de sementes de pinhão-manso, Ciênc. agrotec., Lavras, v. 32, n. 3, p. 863-868, maio/jun., 2008.

NOBRE, R.G.; GHEVI, H.R.; SOARES, F.A.L.; CARDOSO, J.A.F.; Produção de girassol sob estresse salino e adubação nitrogenada, R. Bras. Ci. Solo, 35:929-937, 2011.

PASCUALI L.C.; SILVA F.S.; PORTO A.G.; SILVA FILHO A.; MENEGHELLO G.E. Germinação de sementes de pinhão manso em diferentes temperaturas, luz e substratos Semina: Ciências Agrárias, Londrina, v. 33, n. 4, p. 1435-1440, jul./ago. 2012.

SILVA, J.I.C.; MARTINS, D.; PEREIRA, M.R.R.; RODRIGUES, A.C.P.; COSTA, N.V.; Determinação dos períodos de interferência de plantas daninhas na cultura do girassol, Planta Daninha, Viçosa-MG, v. 30, n. 1, p. 27-36, 2012.

USDA - United States Department of Agriculture - lifting of the grain harvest - December/2013.

ZANELLA, F.; LOPES, P.M.; LIMA, A.L.S. Germinação e crescimento de plântulas de feijão-vagem sob diferentes regimes térmicos. In: Congresso Nacional de Botânica, 2006. Gramado - Rio Grande do Sul. Anais... Gramado, 2006. CDROM. 